\title{
Innovative analysis of distant metastasis in differentiated thyroid cancer
}

\author{
DANYANG CHEN $^{1 *}$, LEI HUANG $^{1 *}$, SICHAO CHEN $^{1 *}$, YIHUI HUANG $^{1}$, DI HU $^{1}$, WEN ZENG $^{2}$, MIN WANG $^{1}$, \\ WEI ZHOU ${ }^{1}$, HAIFENG FENG ${ }^{1}$, WEI WEI ${ }^{3}$, CHAO ZHANG ${ }^{4}$, ZEMING LIU ${ }^{1}$ and LIANG GUO ${ }^{1}$ \\ Departments of ${ }^{1}$ Plastic Surgery and ${ }^{2}$ Ophthalmology, Zhongnan Hospital of Wuhan University, Wuhan, \\ Hubei 430071, P.R. China; ${ }^{3}$ Department of Pediatrics, St. John Hospital and Medical Center, Detroit, \\ MI 48236, USA; ${ }^{4}$ Department of Cardiovascular Surgery, Union Hospital, Tongji Medical College, \\ Huazhong University of Science and Technology, Wuhan, Hubei 430030, P.R. China
}

Received March 24, 2019; Accepted September 17, 2019

DOI: $10.3892 / \mathrm{ol} .2020 .11304$

\begin{abstract}
Differentiated thyroid cancer (DTC) is the most common thyroid cancer with a relatively high survival rate. The association between certain risk factors of distant metastasis (DM) remains uncertain. In order to assess the prognosis of patients with DTC better, the present study aimed to investigate the synergistic effects between histologic subtype, tumor size, lymph node metastasis (LNM) status and extrathyroidal extension (ETE) on the incidence of DM in DTC. Data of 96,788 patients with DTC were obtained from the Surveillance, Epidemiology and End Results database. The association between clinicopathological factors and DM was evaluated by univariate and multivariate analyses. The synergistic effects of relevant factors were determined by measuring the relative excess risk due to interaction (RERI), attributable proportion due to interaction (AP) and synergy index (SI). The results demonstrated that tumor size, LNM status, histologic subtype and ETE were independent risk factors for DM [odds ratio $(\mathrm{OR})=2.433 ; \mathrm{P}<0.001 ; \mathrm{OR}=3.998 ; \mathrm{P}<0.001 ; \mathrm{OR}=6.266$; $\mathrm{P}<0.001$; and $\mathrm{OR}=3.873 ; \mathrm{P}<0.001$, respectively]. In addition, a significant additive synergistic effect on DM was identified between ETE and histologic subtype, ETE and LNM status, as well as between ETE and tumor size (RERI=34.097; $\mathrm{AP}=0.706 ; \mathrm{SI}=3.585 ; \mathrm{RERI}=6.425 ; \mathrm{AP}=0.410 ; \mathrm{SI}=1.781$; and $\mathrm{RERI}=76.973$; $\mathrm{AP}=0.864 ; \mathrm{SI}=7.930$, respectively). Therefore, the results of this study revealed that ETE with follicular thyroid histology, N1 stage or large tumor size may have a
\end{abstract}

Correspondence to: Dr Liang Guo or Dr Zeming Liu, Department of Plastic Surgery, Zhongnan Hospital of Wuhan University, 169 Donghu Road, Wuchang, Wuhan, Hubei 430071, P.R. China

E-mail: guoliangwhzn@163.com

E-mail: 96myt@163.com

${ }^{*}$ Contributed equally

Key words: differentiated thyroid cancer, distant metastasis, synergistic effect, extrathyroidal extension synergistic effect on the risk of DM in patients with DTC. These results suggested that individualized treatment may benefit patients with DTC, and when ETE coexists with one of the identified risk factors, radical treatments may be required.

\section{Introduction}

Thyroid cancer is the most common malignancy of the thyroid, with overall incidence rate of 15.04 per 100,000 individuals reported in 2015 (1). Among the types of thyroid cancer, differentiated thyroid cancer (DTC) accounts for $90-95 \%$ of all cases (2). The major types of DTC are papillary thyroid carcinoma (PTC) and follicular thyroid carcinoma (FTC) (3). Multiple treatments are applied for DTC, including surgical treatment, thyroid-stimulating hormone suppression, radioiodine, molecular-targeted radiation and biological therapy $(4,5)$. The development of individualized, dynamic clinical treatment has greatly improved the survival rate of patients with DTC, with the 10-year survival rate reaching $90 \%$ in U.S over the past 40 years, according to the American Joint Committee on Cancer (AJCC) Cancer Staging Manual (8th edition) (6). However, a number of patients with DTC still exhibit poor survival due to the lack of effective treatment (7).

A high percentage of mortality from DTC is associated with distant metastasis (DM), which is identified at diagnosis in $2-5 \%$ of patients with DTC $(8,9)$. The lung parenchyma is the most common site of DM, followed by the bone, brain, liver, kidney and skin $(10,11)$. Therefore, the detection of DM in patients with DTC at an early stage is important for further improving the survival rate.

According to previous studies, the presence of extrathyroidal extension (ETE) in patients with DTC, especially gross ETE, may be used to evaluate postoperative therapeutic strategies and is associated with the survival of patients with DM $(12,13)$. In addition, the survival rates of patients with PTC and FTC have been reported to be significantly associated with DM (14,15). Lymph node metastasis (LNM) status and tumor size are also associated with DM in patients with PTC $(16,17)$. Previous studies have also identified ETE and LNM status as independent risk factors for DM in patients with DTC (18-20). However, the combined effect of these factors, especially that 
of ETE with the other factors, remains uncertain. Therefore, the present study aimed to investigate the synergistic effect between ETE and tumor size, histologic subtype or LNM status on the incidence of DM in patients with DTC, which may help clinicians to better assess the prognosis of patients and select appropriate treatment strategies.

\section{Materials and methods}

Data collection. The retrospective protocol of this study was approved by the Ethics Committee of Zhongnan Hospital of Wuhan University (Wuhan, China). Patient data were obtained from the Surveillance, Epidemiology, and End Results (SEER) Program of the National Cancer Institute (https://seer.cancer. gov/about/overview.html). The study cohort included patients with a diagnosis of DTC (PTC or FTC) according to the International Classification of Oncological Diseases, 3rd edition (21), as the index (first) diagnosed malignancy. Patients with Hürthle cell thyroid carcinoma, poorly differentiated thyroid carcinoma, anaplastic (undifferentiated) carcinoma, medullary thyroid cancer, thyroid lymphoma, thyroid cancer arising from a thyroglossal duct cyst and thyroid cancer in malignant struma ovarii were excluded from the study. Patients with incomplete follow-up or missing data were also excluded. Data were extracted using the SEER* Stat software (version 8.3.2; Surveillance Research Program, National Cancer Institute; www.seer.cancer.gov/seerstat) and imported into a Microsoft Excel spreadsheet (Microsoft Corporation). Information on clinicopathological characteristics, primary tumor and treatment strategies were obtained from 96,788 patients with DTC between January 2004 and December 2013. Informed consent was obtained from each patient following a full explanation of the purpose and nature of the cancer incidence and survival data used, and patient records were anonymized and de-identified prior to analysis.

Patients were categorized by sex (male or female), ethnicity (white, black, other or unknown) and age at diagnosis $(<55$ or $\geq 55$ years) (22). In addition, patients were categorized by pathological features such as tumor size ( $\leq 10$ or $>10 \mathrm{~mm})$ (22), LNM status (N0 or N1 stage), DM (M0 or M1 phase), multifocality, histologic subtype (PTC or FTC) and presence of ETE. Patients were also grouped according to treatment, including radiation therapy (none or refused, radiation beam/radioactive implants or radioisotopes/radiation beam plus isotopes/implants) and surgery (none, lobectomy, subtotal/near-total thyroidectomy or total thyroidectomy).

Statistical analysis. Data were imported to SPSS (version 21.0; IBM Corp.) for statistical analysis. Patients with DTC were divided into DM and non-DM groups. To assess the independent risk factors affecting DM, clinicopathological characteristics were compared using the $\chi^{2}$ test for univariate analysis; binary logistic regression analysis was performed for the relevant variables (age, sex, ethnicity, tumor size, $\mathrm{N}$ stage, histology subtype, extrathyroidal extension).

The association between DM incidence and risk factors, including tumor size, histologic subtype and ETE, was assessed using logistic regression analysis with adjustment for other cofounding variables. In addition, 95\% confidence intervals (CIs) were calculated precisely. Patients were divided into different groups based on the combination of ETE with the three other factors. The relative excess risk due to interaction (RERI), attributable proportion (AP) due to interaction and synergy index (SI) were calculated to evaluate the synergistic effect of these factors on DM in patients with DTC, where RERI $>0, A P>0$ or $\mathrm{S}>1$ indicated a synergistic effect. No additive synergistic effect was indicated by the $95 \%$ CIs of RERI and $\mathrm{AP}=0$ and $\mathrm{SI}=1$. The Kaplan-Meier method and log-rank test were used to evaluate the influence of the four factors on patient survival.

P-values were two-sided, and $\mathrm{P}<0.05$ was considered to indicate a statistically significant difference. Statistical analyses were performed using SPSS version 21.0 (IBM Corp.), R statistical software (R Core Development Team), and GraphPad Prism version 6 (GraphPad Software, Inc.).

\section{Results}

General characteristics of the study population. Of the 96,788 patients with DTC, $60,841(62.9 \%)$ were aged $<55$ years and 74,325 (76.8\%) were female. Of all patients, 79,063 (82.7\%) were white, $6,299(6.6 \%)$ were black and 10,246 (10.7\%) were of other ethnicities. In addition, 37,584 (39.9\%) patients exhibited multifocality, 5,718 (5.9\%) had FTC, $31,525(34.3 \%)$ exhibited tumors $<10 \mathrm{~mm}, 15,339$ (16.0\%) exhibited ETE, 20,963 (21.9\%) were diagnosed with LNM and 1,488 (1.5\%) exhibited DM. All demographic and clinicopathological characteristics are presented in Table I.

Factors associated with DM in DTC. The results of the univariate analysis (Table II) revealed that patients with DTC with large tumor size $(>10 \mathrm{~mm} ; \mathrm{P}<0.001)$, ETE $(\mathrm{P}<0.001)$ or LNM $(\mathrm{P}<0.001)$ were more likely to develop DM. In addition, compared with patients with PTC, those with FTC exhibited an increased risk of DM $(\mathrm{P}<0.001)$. DM in patients with DTC was also associated with age, sex, ethnicity and multifocality (all $\mathrm{P}<0.001)$.

To further investigate the association between the clinicopathological factors (ETE, histologic subtype, tumor size and LNM status) and DM, multivariate analysis was performed with adjustments for age, sex, and ethnicity. Similar results to univariate analysis were obtained (Table III): Tumor size, LNM status, histologic subtype and ETE were identified as independent risk factors for DM [odds ratio $(\mathrm{OR})=2.433 ; 95 \%$ CI, 1.910-3.098; $\mathrm{P}<0.001$; OR=3.998; 95\% CI, 3.415-4.681; $\mathrm{P}<0.001 ; \mathrm{OR}=6.266 ; 95 \% \mathrm{CI}, 5.176-7.586 ; \mathrm{P}<0.001$; and $\mathrm{OR}=3.873$; 95\% CI, 3.338-4.494; $\mathrm{P}<0.001$, respectively).

Synergistic effects of ETE and histologic subtype, tumor size or LNM status on DM. To comprehensively examine the synergistic effects of ETE and the other clinicopathological factors on DM, all patients were divided into three groups with two factors compared in each group (ETE and histologic subtype, ETE and LNM status and ETE and tumor size).

As presented in Table IV, the probability of DM was the highest in patients with FTC and ETE (OR=26.598; 95\% CI, 19.703-35.906; $\mathrm{P}<0.001)$ compared with the other subgroups. Additionally, the probability of DM was higher in patients with FTC without ETE (OR=5.819; 95\% CI, 4.598-7.363; $\mathrm{P}<0.001)$ and in patients with PTC and ETE $(\mathrm{OR}=3.739 ; 95 \%$ 
Table I. Demographic and clinicopathological characteristics of 96,788 patients with differentiated thyroid carcinoma.

\begin{tabular}{|c|c|}
\hline Characteristics & $\mathrm{N}(\%)$ \\
\hline \multicolumn{2}{|l|}{ Age at diagnosis, years } \\
\hline Mean (range) & $49(2-105)$ \\
\hline$<55$ years & $60,841(62.9)$ \\
\hline$\geq 55$ years & $35,947(37.1)$ \\
\hline \multicolumn{2}{|l|}{ Sex } \\
\hline Female & $74,325(76.8)$ \\
\hline Male & $22,463(23.2)$ \\
\hline \multicolumn{2}{|l|}{ Ethnicity } \\
\hline White & $79,063(82.7)$ \\
\hline Black & $6,299(6.6)$ \\
\hline Other & $10,246(10.7)$ \\
\hline \multicolumn{2}{|l|}{ Tumor size (mm) } \\
\hline Mean (range) & $18.59(0-988)$ \\
\hline$\leq 10 \mathrm{~mm}$ & $31,525(34.3)$ \\
\hline$>10 \mathrm{~mm}$ & $60,451(65.7)$ \\
\hline \multicolumn{2}{|l|}{$\mathrm{N}$ stage } \\
\hline No & $74,693(78.1)$ \\
\hline $\mathrm{N} 1$ & $20,963(21.9)$ \\
\hline Distant metastasis & $1,488(1.5)$ \\
\hline Multifocality & $37,584(39.9)$ \\
\hline \multicolumn{2}{|l|}{ Histologic subtype } \\
\hline PTC & $91,070(94.1)$ \\
\hline FTC & $5,718(5.9)$ \\
\hline Extrathyroidal extension & $15,339(16.0)$ \\
\hline \multicolumn{2}{|l|}{ Radiation } \\
\hline None or refused & $48,030(50.8)$ \\
\hline Radiation beam/radioactive implants & $1,831(1.9)$ \\
\hline $\begin{array}{l}\text { Radioisotopes/radiation beam plus } \\
\text { isotopes/implants }\end{array}$ & $44,761(47.3)$ \\
\hline \multicolumn{2}{|l|}{ Surgery } \\
\hline Lobectomy & $13,723(14.7)$ \\
\hline Subtotal/near-total thyroidectomy & $3,572(3.8)$ \\
\hline Total thyroidectomy & $76,075(81.5)$ \\
\hline
\end{tabular}

FTC, follicular thyroid carcinoma; PTC, papillary thyroid carcinoma; N0, no evidence of locoregional lymph node metastasis; N1, metastasis to regional nodes.

CI, 3.182-4.393; $\mathrm{P}<0.001)$ compared with that in patients with PTC without ETE (Table IV).

Based on these results, the RERI of ETE and histologic subtype was 34.097 (95\% CI, 23.068-45.126), which indicated that the additive synergistic effect of the FTC histologic subtype and ETE contributed a 34.097 relative excess risk on the incidence of DM. The AP was 0.706 (95\% CI, 0.638-0.775), which suggested that $70.6 \%$ of DM cases exposed to the two risk factors may be caused by the synergistic effect. The SI was 3.585 (95\% CI, 2.82-4.556), which suggested the existence of a synergistic effect between the DTC histologic subtype and ETE on the incidence of DM.
In the estimation of the synergistic effect between LNM status and ETE, the probability of DM was the highest in patients with ETE and N1 stage $(\mathrm{OR}=16.097 ; 95 \% \mathrm{CI}$, 13.415-19.317; $\mathrm{P}<0.001)$ compared with the other subgroups (Table V). The probability of DM was higher in patients with only LNM (OR=5.047; 95\% CI, 4.062-6.270; P<0.001) or ETE $(\mathrm{OR}=4.902 ; 95 \% \mathrm{CI}, 3.967-6.059 ; \mathrm{P}<0.001)$ compared with that in patients without ETE and LNM. In addition, a RERI of 6.425 was contributed by the additive synergistic effect of ETE and LNM on the incidence of DM (95\% CI, 4.543-8.307). In addition, $41.0 \%$ of DMs exposed to the two risk factors were likely caused by the additive synergistic effect $(\mathrm{AP}=0.410$; 95\% CI, 0.323-0.498). The SI was 1.781 (95\% CI, 1.511-2.098), suggesting the existence of a synergistic effect between LNM status and ETE on the incidence of DM.

The synergistic effect between ETE and tumor size on the incidence of DM was also evaluated (Table VI). The probability of DM was the highest in patients with large tumors ( $>10 \mathrm{~mm})$ and ETE (OR=9.727; 95\% CI, 7.295-12.969; $\mathrm{P}<0.001)$ compared with those with other combinations of tumor size and ETE. In addition, the probability of DM was significantly higher in patients with larger tumors $(>10 \mathrm{~mm})$ without ETE $(\mathrm{OR}=2.537$; 95\% CI, 1.903-3.383; $\mathrm{P}<0.001)$ and in patients with small tumors $(\leq 10 \mathrm{~mm})$ with $\mathrm{ETE}(\mathrm{OR}=4.430$; 95\% CI, 2.678-7.328; $\mathrm{P}<0.001)$ compared with that in patients with small tumors without ETE.

Further analysis indicated a 76.973(95\% CI,50.238-103.708) relative excess risk contributed by the additive synergistic effect of tumor size and ETE on the incidence of DM. The AP was 0.864 (95\% CI, 0.823-0.905), which suggested that $86.4 \%$ of DM cases exhibiting the two risk factors may have been caused by the synergistic effect. In addition, the SI was 7.930 (95\% CI, 5.833-10.780), indicating a synergistic effect between tumor size and ETE on the incidence of DM.

Synergistic effects of ETE and histologic subtype, tumor size or LNM status on patient survival. The synergistic effects of ETE and tumor size, histologic subtype or LNM status on survival of patients with DTC were evaluated using Kaplan-Meier analysis. Among the four groups categorized according to ETE and histologic subtype (Fig. 1), the cancer-specific survival curves were relatively flat for patients without ETE, whereas patients with PTC and ETE exhibited a modest decline in the survival curve. By contrast, patients with FTC and ETE exhibited a sharp decline in the survival curve. The all-cause survival curve of patients with FTC without ETE was relatively flat, and the survival curve of patients with FTC and ETE exhibited a sharp decline. Patients with PTC with and without ETE exhibited a modest decline in the survival curve (Fig. 1).

Similarly, in the Kaplan-Meier analysis of the four groups categorized according to ETE and LNM status, patients with N1 stage tumors and ETE exhibited a sharp decline in the cancer-specific survival curve and all-cause survival curve, compared with patients with only ETE or N1 stage tumors and patients with N0 stage tumors without ETE (Fig. 2).

In the Kaplan-Meier analysis of the four groups categorized according to ETE and tumor size, the cancer-specific and all-cause survival curves of patients with larger tumors $(>10 \mathrm{~mm})$ and ETE exhibited a decline in survival compared 
Table II. Association between clinicopathological factors and distant metastasis in differentiated thyroid cancer.

\begin{tabular}{|c|c|c|c|c|}
\hline Characteristic & Group & M0 N (\%) (N=95,300) & M1 N (\%) $(\mathrm{N}=1,488)$ & P-value \\
\hline \multirow[t]{3}{*}{ Age, years } & & $49.31 \pm 15.267$ & $60.54 \pm 18.613$ & $<0.001$ \\
\hline & $<55$ & $60,384(99.2)$ & $457(0.8)$ & \\
\hline & $\geq 55$ & $34,916(97.1)$ & $1,031(2.9)$ & \\
\hline \multirow[t]{2}{*}{ Sex } & Female & $73,488(98.9)$ & $837(1.1)$ & $<0.001$ \\
\hline & Male & $21,812(97.1)$ & $651(2.9)$ & \\
\hline \multirow[t]{3}{*}{ Ethnicity } & White & $77,962(98.6)$ & $1,101(1.4)$ & $<0.001$ \\
\hline & Black & $6,160(97.8)$ & $139(2.2)$ & \\
\hline & Other & $10,001(97.6)$ & $245(2.4)$ & \\
\hline \multirow[t]{3}{*}{ Tumor size, mm } & & $18.33 \pm 19.18$ & $38.34 \pm 28.03$ & $<0.001$ \\
\hline & $\leq 10 \mathrm{~mm}$ & $31,387(99.6)$ & $138(0.4)$ & \\
\hline & $>10 \mathrm{~mm}$ & $59,413(98.3)$ & $1,038(1.7)$ & \\
\hline \multirow[t]{2}{*}{$\mathrm{N}$ stage } & N0 & $74,134(99.3)$ & $559(0.7)$ & $<0.001$ \\
\hline & $\mathrm{N} 1$ & $20,210(96.4)$ & $753(3.6)$ & \\
\hline \multirow[t]{2}{*}{ Multifocality } & NO & $55,922(98.9)$ & $626(1.1)$ & $<0.001$ \\
\hline & YES & $37,045(98.6)$ & $539(1.4)$ & \\
\hline \multirow[t]{2}{*}{ Histologic subtype } & PTC & $89,913(98.7)$ & $1,157(1.3)$ & $<0.001$ \\
\hline & FTC & $5,387(94.2)$ & $331(5.8)$ & \\
\hline \multicolumn{5}{|c|}{ Extrathyroidal extension } \\
\hline & No & $79,816(99.4)$ & $511(0.6)$ & $<0.001$ \\
\hline & Yes & $14,593(95.1)$ & 746 (4.9) & \\
\hline
\end{tabular}

FTC, follicular thyroid carcinoma; PTC, papillary thyroid carcinoma; M0, no metastasis; M1, distant metastasis; N0, no evidence of locoregional lymph node metastasis; N1, metastasis to regional nodes.

Table III. Multivariate analysis of distant metastases in differentiated thyroid cancer.

\begin{tabular}{lcr}
\hline Characteristic & OR $(95 \% \mathrm{CI})$ & P-value \\
\hline Age & $3.449(3.001-3.964)$ & $<0.001$ \\
Sex & $1.690(1.479-1.932)$ & $<0.001$ \\
Ethnicity (White) & Reference & $<0.001$ \\
Ethnicity (Black) & $1.457(1.119-1.897)$ & 0.005 \\
Ethnicity (other) & $1.498(1.255-1.787)$ & $<0.001$ \\
Size & $2.433(1.910-3.098)$ & $<0.001$ \\
N stage & $3.998(3.415-4.681)$ & $<0.001$ \\
Histologic subtype & $6.266(5.176-7.586)$ & $<0.001$ \\
Extrathyroidal extension & $3.873(3.338 \sim 4.494)$ & $<0.001$ \\
\hline
\end{tabular}

OR, odds ratio; N0, no evidence of locoregional lymph node metastasis; N1, metastasis to regional nodes.

with those of patients with either factor alone, as well as patients with smaller tumors $(\leq 10 \mathrm{~mm})$ without ETE (Fig. 3).

\section{Discussion}

DTC exhibits a lower mortality rate compared with other carcinomas, but certain types of DTC, such as radioiodine refractory differentiated thyroid carcinoma, are difficult to diagnose at an early stage $(23,24)$. Patients with DTC have poor prognosis due to DM (25-27). Previous studies have indicated that LNM, tumor size, histologic subtype and ETE are risk factors that influence the prognosis of patients with DTC $(20,28,29)$. However, the majority of these studies were single-center, small-sample studies or meta-analyses, and the synergistic effects of these risk factors have not been sufficiently elaborated.

The SEER database contains data encompassing approximately $28 \%$ of the US population (30). SEER registries hold the highest level of certification of data quality provided by the North American Association of Central Cancer Registries (31). In addition, the index of completeness of case ascertainment of the program is $98 \%$, making it the gold standard database for cancer analysis in the US (32-34). Therefore, the SEER database allows in-depth analysis of certain clinical issues.

The presence of DM is considered to be a significant factor at diagnosis and during staging according to the Tumor-Node-Metastasis (TNM) classification $(35,36)$, and a confirmed diagnosis of DM is associated with mortality or poor prognosis in patients with FTC and PTC (37). Predicting the incidence of DM in patients with DTC may help develop an appropriate treatment strategy. The treatment for patients with DTC without DM includes local therapeutic measures, such as surgery and radiofrequency; however, in patients with DM, radioiodine therapy is considered the most effective treatment (38). Therefore, predicting the most unfavorable prognostic factors for DM is crucial for determining treatment strategies for patients with DTC.

The present study used data from patients with DTC and DM from the SEER database to evaluate the risk factors and 
Table IV. Measures for estimation of synergistic effect between histologic subtype and ETE for the risk of distant metastasis in differentiated thyroid cancer.

\begin{tabular}{lcccc}
\hline Subtype & ETE & M1 cases $(\%)$ & Total cases & OR $(95 \%$ CI $)$ \\
\hline PTC & No & $368(0.5)$ & 75,278 & Reference \\
FTC & No & $143(2.8)$ & 5,049 & $5.819(4.598-7.363)$ \\
PTC & Yes & $644(4.3)$ & 14,807 & $3.739(3.182-4.393)$ \\
FTC & Yes & $102(19.2)$ & 532 & $26.598(19.703-35.906)$ \\
RERI & & & $34.097(23.068-45.126)$ & $<0.001$ \\
AP & & & $0.706(0.638-0.775)$ & $<0.001$ \\
SI & & & $3.585(2.82-4.556)$ \\
\hline
\end{tabular}

ETE, extrathyroidal extension; FTC, follicular thyroid carcinoma; PTC, papillary thyroid carcinoma; DTC, differentiated thyroid cancer; OR, odds ratio; RERI, relative excess risk due to interaction; AP, attributable proportion due to interaction; SI, synergy index; M1, distant metastasis.

Table V. Measures for estimation of synergistic effect between $\mathrm{N}$ stage and ETE for the risk of distant metastasis in differentiated thyroid cancer.

\begin{tabular}{|c|c|c|c|c|c|}
\hline $\mathrm{N}$ stage & ETE & M1 cases & Total cases & OR $(95 \% \mathrm{CI})$ & P-value \\
\hline 0 & No & 285 & 67,262 & Reference & \\
\hline 1 & No & 189 & 12,406 & $5.047(4.062-6.270)$ & $<0.001$ \\
\hline 0 & Yes & 191 & 7,000 & $4.902(3.967-6.059)$ & $<0.001$ \\
\hline 1 & Yes & 509 & 8,151 & $16.097(13.415-19.317)$ & $<0.001$ \\
\hline RERI & & \multicolumn{4}{|c|}{$6.425(4.543-8.307)$} \\
\hline AP & & \multicolumn{4}{|c|}{$0.410(0.323-0.498)$} \\
\hline SI & & \multicolumn{4}{|c|}{$1.781(1.511-2.098)$} \\
\hline
\end{tabular}

ETE, extrathyroidal extension; OR, odds ratio; RERI, relative excess risk due to interaction; AP, attributable proportion due to interaction; SI, synergy index; M1, distant metastasis; N0, no evidence of locoregional lymph node metastasis; N1, metastasis to regional nodes.

Table VI. Measures for estimation of synergistic effect between tumor size and ETE for the risk of distant metastasis in differentiated thyroid cancer.

\begin{tabular}{lcccc}
\hline Tumor size & ETE & M1 cases & Total cases & OR (95\% CI) \\
\hline$\leq 10 \mathrm{~mm}$ & No & 63 & 29,774 & Reference \\
$>10 \mathrm{~mm}$ & No & 388 & 47,029 & $2.537(1.903-3.383)$ \\
$\leq 10 \mathrm{~mm}$ & Yes & 27 & 1,518 & $4.430(2.678-7.328)$ \\
$>10 \mathrm{~mm}$ & Yes & 613 & 13,204 & $9.727(7.295-12.969)$ \\
RERI & & & $76.973(50.238-103.708)$ & $<0.001$ \\
AP & & & $0.864(0.823-0.905)$ & $<0.001$ \\
SI & & & $7.930(5.833-10.780)$ \\
\hline
\end{tabular}

ETE, extrathyroidal extension; OR, odds ratio; RERI, relative excess risk due to interaction; AP, attributable proportion due to interaction; SI, synergy index; M1, distant metastasis.

analyze the synergistic effects of ETE and the other risk factors. The results of the univariate and multivariate analyses determined that ETE, histologic subtype, tumor size and LNM status were independent factors that influenced the incidence of DM. Among these independent factors, ETE has been emphasized in previous studies. The American Joint Committee on
Cancer (AJCC) Cancer Staging Manual (8th edition) (35) has reported that gross ETE may be identified clearly as T3b, T4a, or T4b based on imaging or intraoperative findings. In addition, due to the poor survival outcomes associated with gross ETE, its presence is considered to be a significant reference index for the staging category $(35,39)$. Thus, ETE was selected 

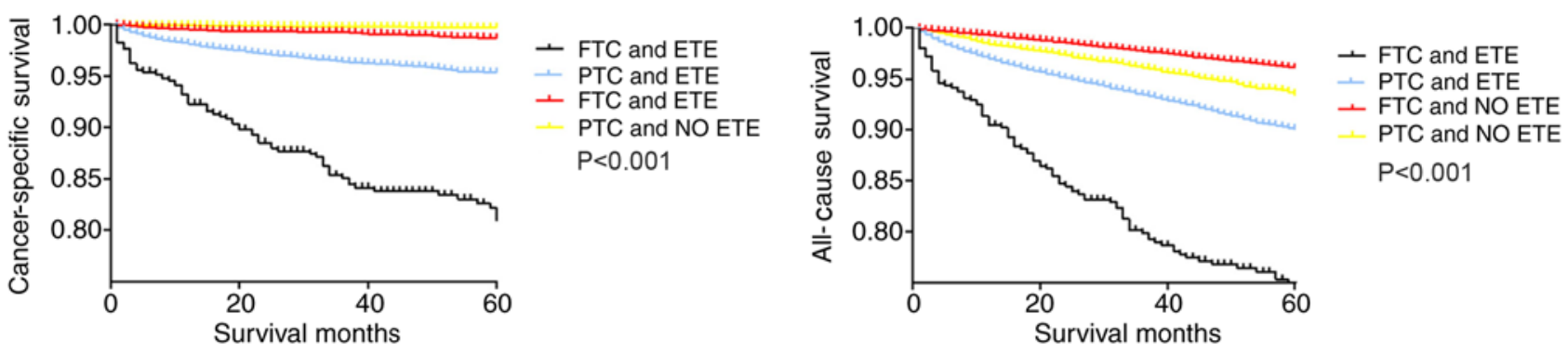

Figure 1. Kaplan Meier curves of cancer-specific survival and all-cause survival. Effects of histologic subtype and ETE on the survival of patients with differentiated thyroid cancer. FTC, follicular thyroid carcinoma; PTC, papillary thyroid carcinoma; ETE, extrathyroidal extension.
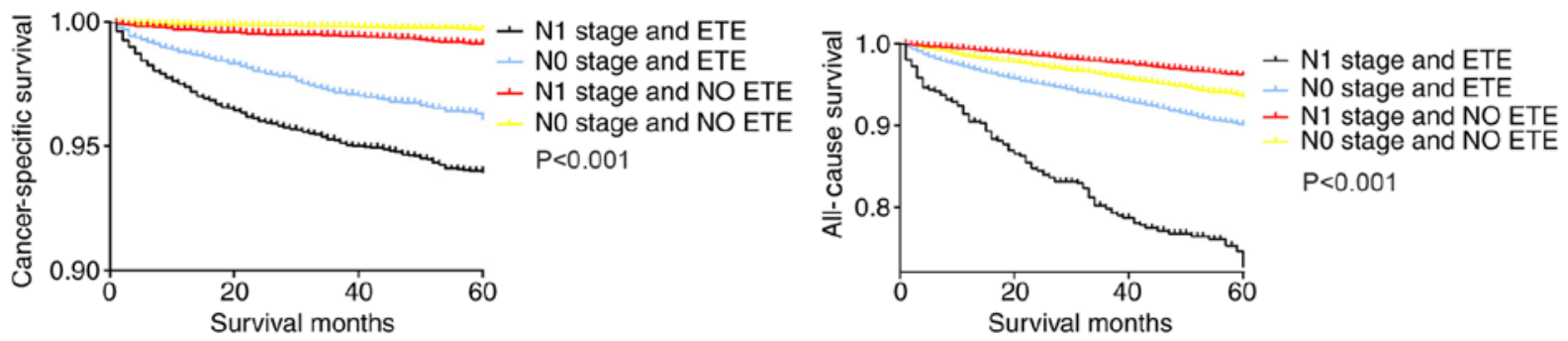

Figure 2. Kaplan Meier curves of cancer-specific survival and all-cause survival. Effects of N stage and ETE on the survival of patients with differentiated thyroid cancer. ETE, extrathyroidal extension; N0, no evidence of locoregional lymph node metastasis; N1, metastasis to regional nodes.
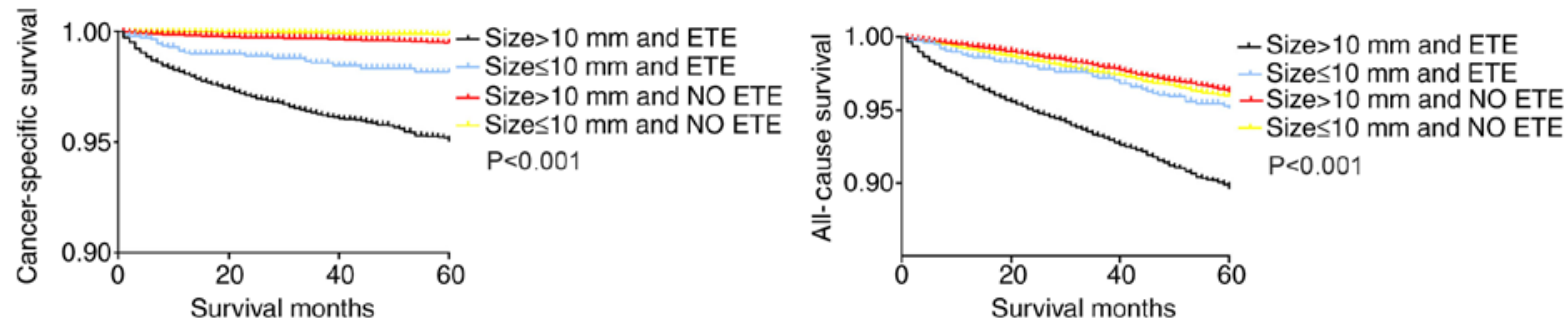

Figure 3. Kaplan Meier curves for cancer-specific survival and all-cause survival. Effects of tumor size and ETE on the survival of patients with differentiated thyroid cancer. ETE, extrathyroidal extension.

as the key factor in the present study, and its interaction with the three other factors was investigated.

The preliminary analyses of the present study demonstrated that patients with FTC and ETE obtained the highest incidence of DM compared with the other subgroups (patients with FTC and ETE, patients with FTC without ETE, and patients with PTC and ETE). Patients with ETE and large tumor size exhibited the highest incidence of DM. Patients with ETE and LNM also exhibited a higher incidence of DM compared with the other subgroups. Therefore, the results of the present study suggest synergistic effects of ETE and histologic subtype, LNM status or tumor size on the incidence of DM in patients with DTC. Furthermore, the analyses of survival curves using the Kaplan-Meier method also demonstrated synergistic effects of ETE and the other risk factors on survival; a similar tendency was observed in both cancer-specific and all-cause survival

More specifically, the evaluation of the synergistic effects based on RERI, AP and SI indicated that the FTC histologic subtype, large tumor size or presence of LNM may contribute an added risk of DM to patients with DTC who also exhibit ETE.
However, there were several limitations to this study that need to be addressed. First, the study was retrospective, and thus, selection bias was inevitable. Second, in the 8th edition of the TNM/AJCC classification system, ETE was classified into minor ETE and gross ETE, and only gross ETE was considered as a risk factor for staging since gross extrathyroidal extension can be identified clearly by imaging or intraoperative findings, whereas minor ETE is difficult to identify $(13,35)$. Thus, more data containing gross ETE, and not minor ETE, should be included in future research. Third, all data on ETE from the SEER database were based on pathological diagnosis. However, the majority of the data on ETE in clinic are provided by ultrasonologists during diagnosis. Therefore, in clinical application, the conclusion of this study may be subjected to the subjective influence of ultrasonologists and may vary by institution or provider (40). In addition, the SEER database may contain certain bias resulting from the sole inclusion of patients registered with Medicare, with data based only on diagnoses or procedures covered by the insurance.

The synergistic effects of histologic subtype, tumor size, ETE and LNM status on DM might benefit cancer 
management; if two of the four variables are present, this may suggest that the patients with DTC may be more likely to exhibit DM, which is difficult to diagnose at an early stage. Thus, aggressive treatment may be recommended, such as surgical treatment, thyroid-stimulating hormone suppression, radioiodine, molecular targeted or biological therapy $(4,5)$.

In summary, histologic subtype, tumor size, ETE and LNM status were identified in the present study as independent risk factors of DM in patients with DTC. The presence of ETE and FTC, ETE and large tumor size or ETE and LNM may have a synergistic effect of increasing the risk on DM in patients with DTC. Study of the associations between these parameters and DM may be helpful for clinicians for predicting prognosis and developing appropriate treatment strategies. To improve data accuracy, more precise evaluations can be performed in patients with DTC by including more risk factors in the analysis. DM, as a significant independent factor for survival, may also have synergistic effects with other associated factors. This may provide guidance for further research on the associations among demographic factors in the future.

\section{Acknowledgements}

Not applicable.

\section{Funding}

No funding was received.

\section{Authors' contributions}

ZML, LG conceived the idea and designed the study. DYC, LH, YHH, DH, WZe, MW, WZh and HFF undertook the data collection and prepared the tables. DYC, LH, WW and CZ performed the data analysis. DYC, LH, SCC contributed to the interpretation of data and prepared the figures. LH, DYC, LG drafted the manuscript. All authors revised the manuscript critically and approved the final version.

\section{Availability of data and materials}

The datasets used or analyzed during the current study are available from the corresponding author on reasonable request.

\section{Ethics approval and consent to participate}

This study's retrospective protocol was approved by the Ethics Committee of Zhongnan Hospital of Wuhan University. Informed consent has been obtained from each patient after full explanation of the purpose and nature of all procedures used, and patient records were anonymized and de-identified prior to the analysis.

\section{Patient consent for publication}

Not applicable.

\section{Competing interests}

The authors declare that they have no competing interests.

\section{References}

1. Howlader N, Noone AM, Krapcho M, Miller D, Bishop K, Kosary CL, Yu M, Ruhl J, Tatalovich Z, Mariotto A (eds), et al: SEER Cancer Statistics Review, 1975-2014. National Cancer Institute, Bethesda, MD, April 2017.

2. Fardella C, Jimenez M, Gonzalez H, León A, Goñi I, Cruz F, Solar A, Torres J, Mosso L, González G, et al: Pathological characteristics of thyroid microcarcinoma. A review of 402 biopsies. Rev Med Chil 133: 1305-1310, 2005 (In Spanish).

3. Galuppini F, Vianello F, Censi S, Barollo S4, Bertazza L, Carducci S, Colato C, Manso J, Rugge M, Iacobone M, et al: Differentiated thyroid carcinoma in pediatric age: Genetic and clinical scenario. Front Endocrinol (Lausanne) 10: 552, 2019.

4. Zanotti-Fregonara P, Hindie E, Faugeron I, Moretti JL, Ravasi L, Rubello D and Toubert ME: Update on the diagnosis and therapy of distant metastases of differentiated thyroid carcinoma. Minerva Endocrinol 33: 313-327, 2008.

5. Tuttle RM, Ball DW, Byrd D, Dilawari RA, Doherty GM, Duh QY, Ehya H, Farrar WB, Haddad RI, andeel F, et al: Thyroid carcinoma. J Natl Compr Canc Netw 8: 1228-1274, 2010.

6. Shin JJ and Milas M: Detection of disease recurrence in differentiated thyroid cancer. Minerva Chir 65: 101-116, 2010.

7. Goffredo P, Sosa JA and Roman SA: Differentiated thyroid cancer presenting with distant metastases: A population analysis over two decades. World J Surg 37: 1599-1605, 2013.

8. Mazzaferri EL and Kloos RT: Clinical review 128: Current approaches to primary therapy for papillary and follicular thyroid cancer. J Clin Endocrinol Metab 86: 1447-1463, 2001.

9. Hay ID: Papillary thyroid carcinoma. Endocrinol Metab Clin North Am 19: 545-576, 1990.

10. Schlumberger M, Challeton C, De Vathaire F, Travagli JP, Gardet P, Lumbroso JD, Francese C, Fontaine F, Ricard M and Parmentier C: Radioactive iodine treatment and external radiotherapy for lung and bone metastases from thyroid carcinoma. J Nucl Med 37: 598-605, 1996.

11. Toubert ME, Hindie E, Rampin L, Al-Nahhas A and Rubello D: Distant metastases of differentiated thyroid cancer: Diagnosis, treatment and outcome. Nucl Med Rev Cent East Eur 10: 106-109, 2007.

12. Andersen PE, Kinsella J, Loree TR, Shaha AR and Shah JP: Differentiated carcinoma of the thyroid with extrathyroidal extension. Am J Surg 170: 467-470, 1995.

13. Park SY, Kim HI, Kim JH, Kim JS, Oh YL, Kim SW, Chung JH, Jang HW and Kim TH: Prognostic significance of gross extrathyroidal extension invading only strap muscles in differentiated thyroid carcinoma. Br J Surg 105: 1155-1162, 2018.

14. Kim H, Shin JH, Hahn SY, Oh YL, Kim SW, Park KW and Lim Y: Prediction of follicular thyroid carcinoma associated with distant metastasis in the preoperative and postoperative model. Head Neck 41: 2507-2513, 2019.

15. Aboelnaga EM and Ahmed RA: Difference between papillary and follicular thyroid carcinoma outcomes: An experience from Egyptian institution. Cancer Biol Med 12: 53-59, 2015.

16. Mizukami Y, Michigishi T, Nonomura A, Hashimoto T, Terahata S, Noguchi M, Hisada K and Matsubara F: Distant metastases in differentiated thyroid carcinomas: A clinical and pathologic study. Hum Pathol 21: 283-290, 1990.

17. Barbosa MP, Momesso D, Bulzico DA, Farias T, Dias F, Lima RA, Corbo R, Vaisman M and Vaisman F: Metastatic lymph node characteristics as predictors of recurrence/persistence in the neck and distant metastases in differentiated thyroid cancer. Arch Endocrinol Metab 61: 584-589, 2017.

18. Mercante G, Frasoldati A, Pedroni C, Formisano D, Renna L, Piana S, Gardini G, Valcavi R and Barbieri V: Prognostic factors affecting neck lymph node recurrence and distant metastasis in papillary microcarcinoma of the thyroid: Results of a study in 445 patients. Thyroid 19: 707-716, 2009.

19. Jeon MJ, Kim WG, Kim TH, Kim HK, Kim BH, Yi HS, Kim ES, Kim H, Kim YN, Kim EH, et al: Disease-Specific mortality of differentiated thyroid cancer patients in Korea: A Multicenter Cohort Study. Endocrinol Metab (Seoul) 32: 434-441, 2017

20. Vuong HG, Duong UNP, Pham TQ, Tran HM, Oishi N, Mochizuki K, Nakazawa T, Hassell L, Katoh R and Kondo T: Clinicopathological risk factors for distant metastasis in differentiated thyroid carcinoma: A meta-analysis. World J Surg 42: 1005-1017, 2018

21. Fritz A,Percy C,Jack A, Shanmugaratnam K, Sobin L, Parkin DM and Whelan S: Third edition, World Health Organization: International Classification of Diseases for Oncology, 2001. 
22. Haugen BR, Alexander EK, Bible KC, Doherty GM, Mandel SJ, Nikiforov YE, Pacini F, Randolph GW, Sawka AM, Schlumberger M, et al: 2015 American thyroid association management guidelines for adult patients with thyroid nodules and differentiated thyroid cancer: The American Thyroid Association guidelines task force on thyroid nodules and differentiated thyroid cancer. Thyroid 26: 1-133, 2016.

23. Kim M, Kim TH, Shin DY,Lim DJ, Kim EY, Kim WB, Chung JH, Shong YK, Kim BH, Kim WG, et al: Tertiary care experience of sorafenib in the treatment of progressive radioiodine-refractory differentiated thyroid carcinoma: A Korean multicenter study. Thyroid 28: 340-348, 2018.

24. de la Fouchardiere C, Alghuzlan A, Bardet S, Borget I, Borson Chazot F, Do Cao C, Godbert Y, Leenhardt L, Zerdoud S and Leboulleux S: The medical treatment of radioiodine-refractory differentiated thyroid cancers in 2019. A TUTHYREF ${ }^{\circledR}$ network review. Bull Cancer 106: 812-819, 2019.

25. Mao Y and Xing M: Recent incidences and differential trends of thyroid cancer in the USA. Endocr Relat Cancer 23: 313-322, 2016.

26. Jeon MJ, Kim WG, Choi YM, Kwon H, Lee YM, Sung TY, Yoon JH, Chung KW, Hong SJ, Kim TY, et al: Features predictive of distant metastasis in papillary thyroid microcarcinomas. Thyroid 26: 161-168, 2016.

27. Nixon IJ, Whitcher MM, Palmer FL, Tuttle RM, Shaha AR, Shah JP, Patel SG and Ganly I: The impact of distant metastases at presentation on prognosis in patients with differentiated carcinoma of the thyroid gland. Thyroid 22: 884-889, 2012.

28. Ito Y, Tomoda C, Uruno T, Takamura Y, Miya A, Kobayashi K, Matsuzuka F, Kuma K and Miyauchi A: Prognostic significance of extrathyroid extension of papillary thyroid carcinoma: Massive but not minimal extension affects the relapse-free survival. World J Surg 30: 780-786, 2006.

29. Bellantone R, Lombardi CP, Boscherini M, Ferrante A, Raffaelli M, Rubino F, Bossola M and Crucitti F: Prognostic factors in differentiated thyroid carcinoma: A multivariate analysis of 234 consecutive patients. J Surg Oncol 68: 237-241, 1998

30. Jacobs AJ, Morris CD and Levin AS: Synovial Sarcoma is not Associated with a higher risk of lymph node metastasis compared with other soft tissue sarcomas. Clin Orthop Relat Res 476: 589-598, 2018
31. Warren JL, Klabunde CN, Schrag D, Bach PB and Riley GF: Overview of the SEER-Medicare data: Content, research applications, and generalizability to the United States elderly population. Med Care 40 (Suppl 8): IV-3-IV18, 2002.

32. Liu Y, Liu Z, Zhao Q, Hua T, Chi S, Huang T and Wang $H$ Propensity score matching analysis of the prognosis for the rare insular subtype of thyroid cancer based on SEER database. Oncotarget 8: 101623-101633, 2017.

33. Liu C, Chen T, Zeng W, Wang S, Xiong Y, Liu Z and Huang T: Reevaluating the prognostic significance of male gender for papillary thyroid carcinoma and microcarcinoma: A SEER database analysis. Sci Rep 7: 11412, 2017.

34. Xiong Y, Zhao Q, Liu C, Wang S, Liu Z and Huang T: Prognosis of patients with TX stage differentiated thyroid cancer: Propensity scored matching analysis of the SEER database 2004-2013. Am J Transl Res 10: 2004-2014, 2018.

35. Amin MB, Edge S, Greene F, Byrd DR, Brookland RK, Washington MK, Gershenwald JE, Compton CC, Hess KR, Sullivan DC, et al: AJCC cancer staging manual. 8th edition. Vol. Springer International Publishing, Chicago, 2017.

36. Tam S, Boonsripitayanon M, Amit M, Fellman BM, Li Y, Busaidy NL, Cabanillas ME, Dadu R, Sherman S, Waguespack SG, et al: Survival in differentiated thyroid cancer: Comparing the AJCC cancer staging seventh and eighth editions. Thyroid 28: 1301-1310, 2018

37. de Melo TG, Zantut-Wittmann DE, Ficher E and da Assumpcao LV: Factors related to mortality in patients with papillary and follicular thyroid cancer in long-term follow-up. J Endocrinol Invest 37: 1195-1200, 2014.

38. Kreissl MC, Janssen MJR and Nagarajah J: Current treatment strategies in metastasized differentiated thyroid cancer. J Nucl Med 60: 9-15, 2019.

39. Shteinshnaider M, Muallem Kalmovich L, Koren S, Or K, Cantrell D and Benbassat C: Reassessment of differentiated thyroid cancer patients using the eighth TNM/AJCC classification system: A Comparative Study. Thyroid 28: 201-209, 2018

40. Kuo EJ, Thi WJ, Zheng F, Zanocco KA, Livhits MJ and Yeh MW: Individualizing surgery in papillary thyroid carcinoma based on a detailed sonographic assessment of extrathyroidal extension. Thyroid 27: 1544-1549, 2017. 